\title{
Protection measures against SARS-CoV-2 infection for cytopathology and histopathology laboratories personnel: practical recommendations
}

\author{
AnCA SAVA ${ }^{1,2)}$, Claudia Florida COSTEA ${ }^{3,4)}$, Şerban TURLIUC ${ }^{5,6)}$, LUCIAN Eva ${ }^{77}$, \\ MiHAELA DANA TURLIUC ${ }^{3,7)}$, GABRIELA FLORENTSA DUMITRESCU²), GABRIELA DimitRIU4), \\ VALERIANA VORNICU ${ }^{2)}$, LILIANA BALTAG ${ }^{8}$, , MARIUS GABRIEL DABIJA ${ }^{3,9)}$ \\ 1) Department of Morpho-Functional Sciences I, Faculty of Medicine, Grigore T. Popa University of Medicine and Pharmacy, \\ Iaşi, Romania \\ 2) Laboratory of Pathology, Prof. Dr. Nicolae Oblu Emergency Clinical Hospital, Iaşi, Romania \\ 3) Department of Surgery II, Faculty of Medicine, Grigore T. Popa University of Medicine and Pharmacy, Iaşi, Romania \\ 4) $2^{\text {nd }}$ Ophthalmology Clinic, Prof. Dr. Nicolae Oblu Emergency Clinical Hospital, Iaşi, Romania \\ 5) III Medical Department, Faculty of Medicine, Grigore T. Popa University of Medicine and Pharmacy, Iaşi, Romania \\ 6) Socola Institute of Psychiatry, Iaşi, Romania \\ 7) $2^{\text {nd }}$ Neurosurgery Clinic, Prof. Dr. Nicolae Oblu Emergency Clinical Hospital, Iaşi, Romania \\ 8) Medical Laboratory, Prof. Dr. Nicolae Oblu Emergency Clinical Hospital, Iaşi, Romania

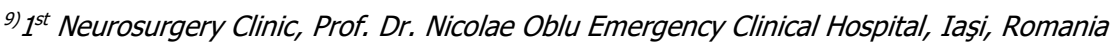

\begin{abstract}
In the severe acute respiratory syndrome coronavirus 2 (SARS-CoV-2) pandemic, healthcare workers are at high risk to be infected with this new coronavirus, particularly when they handle not only patients, but also their body fluids. In Romania, even though the protective measures to be used by medical staff in emergency departments, clinical departments, radiology departments, clinical laboratories and morgues services are well known, there is little information about the protection of medical staff in the laboratories of cytopathology and histopathology. In this article, we will discuss the transmission routes of the new coronavirus, the surfaces it could contaminate in a hospital, as well as the modalities of its inactivation. We will present some guidelines for preparing the pathology departments to face the pandemic situation like the present one. Also, we will point out some possible recommendations/suggestions for protective measures to be taken by laboratory staff during the cytological and histopathological procedures when they manipulate body fluids or surgical samples of patients with suspected or confirmed coronavirus disease 2019 (COVID-19). Laboratory personnel should be aware that any body fluid or surgical specimen that arrives in the laboratory may contain SARS-CoV-2 and, as such, they should act after new working procedures. We recommend restraint from performing extemporaneous examination (smear and frozen section) and cytopathological examination in laboratories that do not have adequate condition for handling and processing Hazard Group 3 (HG3) pathogens, as SARS-CoV-2. Also, laboratory personnel should pay attention to instruments, technical equipment, or environmental surfaces as these also can be contaminated with the new coronavirus.
\end{abstract}

Keywords: SARS-CoV-2 infection, safe handling and processing, surgical specimens, cytopathology and histopathology activities, laboratory personnel.

\section{口 Introduction}

In the last three decades, three pandemics with coronavirus have been noted, which have had an almost curious frequency, respectively every nine years. The first started in the province of Guangdong (China), and then spread to many countries in Southeast Asia, North America, Europe, and South Africa, in a period of almost one year (between November 2002 and September 2003) [1]. The etiological agent was named severe acute respiratory syndrome coronavirus (SARS-CoV) because the patients had fever and severe acute respiratory syndrome. There were 8000 positive cases and 774 deaths worldwide, representing a mortality rate of almost $10 \%$. The second was recorded in 2011. As the disease appeared in the Middle East and manifested by severe acute respiratory distress, its etiological agent, a new coronavirus, received the name MERS-CoV. During a period of six years (from 1 January 2012 to 17 January 2018), a total of 2143 positive cases of MERS-CoV were reported, from 27 countries around the world [2]. In this second pandemic, the number of infected cases was lower, but there were at least 750 deaths, which means that the case fatality rate was much higher (almost 35\%).

In the last days of December 2019, a number of patients with pneumonia of unknown etiology were identified in Wuhan, the capital city of Hubei province (China). After a week, on January 7, 2020, a new coronavirus was isolated from these patients' samples, being temporarily named 2019 novel coronavirus (2019-

This is an open-access article distributed under the terms of a Creative Commons Attribution-NonCommercial-ShareAlike 4.0 International Public License, which permits unrestricted use, adaptation, distribution and reproduction in any medium, non-commercially, provided the new creations are licensed under identical terms as the original work and the original work is properly cited. 
nCoV) by the World Health Organization (WHO) [1] After a month, on February 11, 2020, the virus was subsequently renamed as severe acute respiratory syndrome coronavirus 2 (SARS-CoV-2) and the diseases provoked by this etiological agent received the name coronavirus disease 2019 (COVID-19) by the WHO [3]. However, SARS-CoV-2 has infected until now many more people than either of its two predecessors [4]. At least until the end of April 2020, its mortality rate seems to be smaller than that caused by MERS-CoV, being somewhat similar to that determined by SARS-CoV [3,5] (Figure 1, a-c), but even though SARS-CoV causes similar symptoms through a similar mechanism, there are some differences with the first two pandemics.
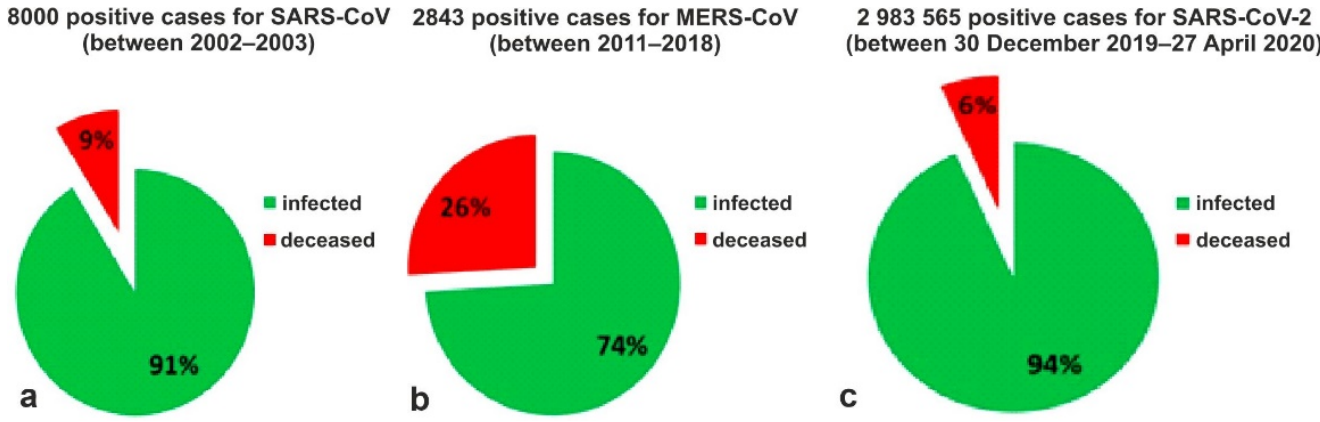

Figure 1 - (a-c) Graphic comparison of mortality rate of all three major coronavirus pandemics, 26 April 2020 (after [3, 5]). MERS-CoV: Middle East respiratory syndrome coronavirus; SARS-CoV-2: Severe acute respiratory syndrome coronavirus 2.

SARS-CoV-2 pandemic is much more important because of its magnitude, as there are an extremely large number of diseases, its spread is much faster, in part due to globalization because the city of Wuhan, a city of 14 million inhabitants, was a large hub connecting the North, South, East and West of China via railways and a major international airport [6] that connected China with all the world. Facing with a growing number of cases, the $W H O$ had to declare the state of pandemic on 11 March 2020, as COVID-19 is affecting 210 countries and territories around the world.

On April 27, 2020, the total number of positive cases for SARS-CoV-2 was over 3000000 worldwide, and the number of those who lost the battle with this microscopic enemy was over 200000 people. If initially everyone saw the epicenter of the pandemic in China, later it moved to Europe, especially in its Western part, where Spain, Italy, France, and United Kingdom, each announced about 200000 positive cases and over 20000 deaths due to this terrible disease. The epicenter of the pandemic has moved to the United States, with more than 20000000 positive cases and more than 400000 deaths, signifying a mortality rate of about $2 \%$.

In Romania, the number of 600000 positive cases has been exceeded and mortality was just over 17000 deaths, which represents a mortality rate of almost $3 \%$ even though rapid measures of social distancing, rapid isolation of cases, correct implementation of protection measures has been taken.

Healthcare workers are at high risk to be infected with SARS-CoV-2, particularly when they handle not only patients, but also their fluids, secretions or blood [7]. In Romania, even though the protective measures to be used by medical staff in emergency departments, clinical departments, radiology department, clinical laboratory and morgues services are well known, there is little information about the protection of medical staff in the laboratories of cytopathology and histopathology. As a result, there is a great need for information regarding the protective measures that must be taken by medical staff of pathology laboratories because they handle biological samples taken from the suspected or confirmed COVID19 patients and this virus is considered to be a Hazard Group 3 (HG3) pathogen ("a biological agent that can cause severe human disease and presents a serious hazard to employees and which may present a risk of spreading to the community, though there is usually effective prophylaxis or treatment available") [8].

\section{Aim}

In this article, we will discuss possible recommendations/ suggestions for protective measures to be taken by laboratory staff during the cytological and histopathological (HP) procedures when they manipulate surgical samples of patients with suspected or confirmed COVID-19.

\section{a How is SARS-CoV-2 transmitted?}

Given the current epidemiological situation (pandemic with the new coronavirus), any patient who undergoes surgery is suspected of being a SARS-CoV2 carrier or may even be infected with this virus.

SARS-CoV-2 may be present not only in biological samples collected from confirmed COVID-19 patients, but also in those collected from patients without a known COVID-19, as they may still be undiagnosed, presymptomatic, asymptomatic, minimally symptomatic or even from convalescent patients [4, 9-11].

It is already well established that SARS-CoV-2 has airborne transmission via aerosols and droplets and that it causes primarily respiratory infections, but now there are more and more studies announcing new routes of transmission.

European Centre for Disease Prevention and Control (ECDC) announced that viral ribonucleic acid (RNA) can persist over long periods of time in body fluids (Figures 2 and 3). SARS-CoV-2 could be identified in respiratory tract specimens (nasopharyngeal swab, oropharyngeal swab, tracheal aspirate, and broncho-alveolar lavage) 1-2 days before the onset of symptoms and it can persist in the respiratory tract for 7-12 days in moderate cases and up to two weeks in severe cases [12]. 


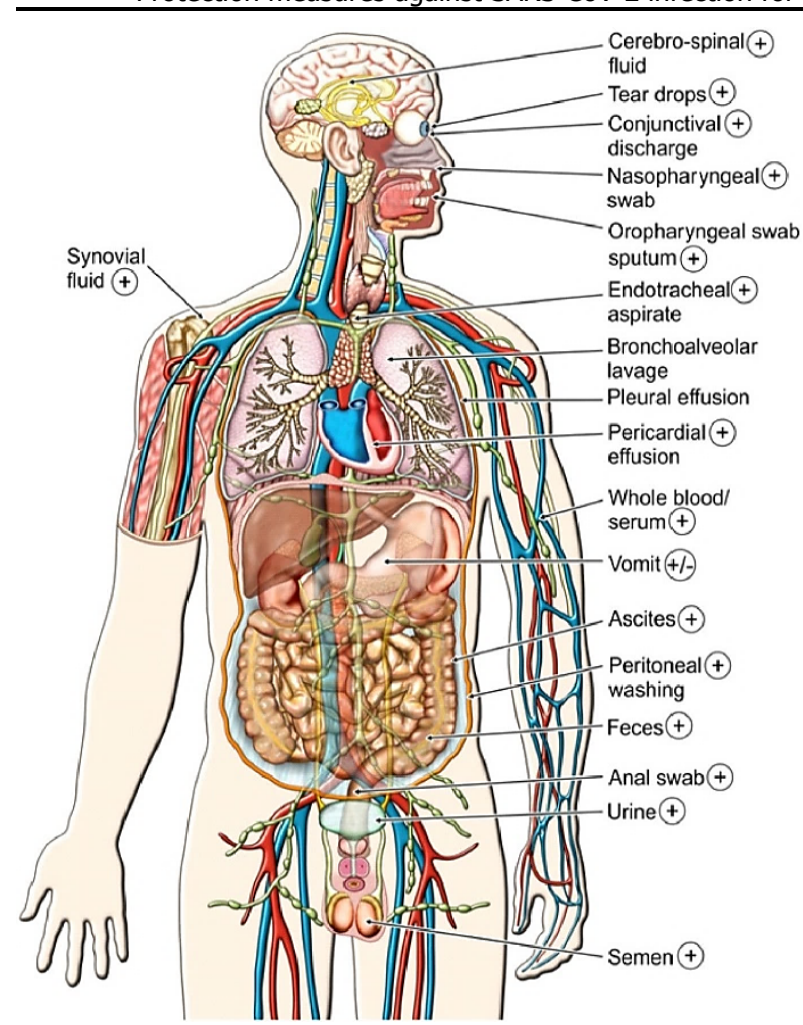

Figure 2 - Respiratory and non-respiratory body fluids, secretions and discharges from SARS-CoV-2 infected persons (man) that could be contain the virus (according to $[10-14,16])$.

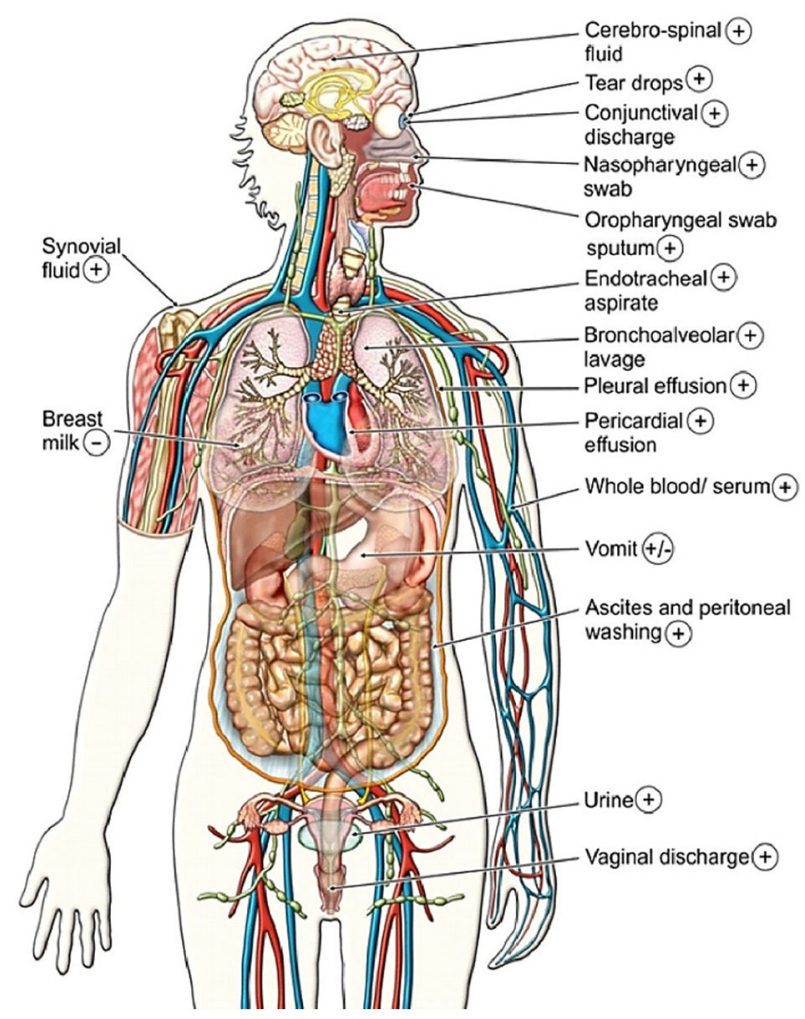

Figure 3 - Respiratory and non-respiratory body fluids, secretions and discharges from SARS-CoV-2 infected persons (woman) that could be contain the virus (according to $[10-14,16])$.

But there are also extra-respiratory routes for virus transmission. In feces, prolonged viral RNA shedding was detected a few days after the onset of the disease and then remained positive for more than one month after infection especially in pediatric patients [11], meaning that SARS-CoV-2 may be transmitted by the fecal route.

SARS-CoV-2 RNA was also detected in urine [13], tears and eye secretions of patients with COVID-19 [10, 14].

A group of researchers from China used quantitative real-time polymerase chain reaction (PCR) to detect the presence of SARS-CoV-2 RNA in body fluids of patients with confirmed SARS-CoV-2 infection. They found that urine and blood specimens, and anal and oropharyngeal swabs contained SARS-CoV-2 RNA, but not all specimen types were positive simultaneously in the same patient [15].

Also, a recent article reported that SARS-CoV-2 RNA was detected in the saliva of infected patients [16].

Salehi et al. (2019), from the University of Southern California, realized a systematic review about COVID-19 and found out that SARS-CoV-2 was also identified in pleural effusion and pericardial effusion, but only in few cases. As such, SARS-CoV-2 is considered an uncommon presence in these two body fluids, but high evidence appeared with disease progression [17].

In COVID-19 patients, SARS-CoV-2 RNA was also detected in plasma, serum or lymphocytes on the first two or three days after onset of symptoms [3], in gastrointestinal tissue $[14,18]$, but not in the placenta from the pregnant women nor in their neonates, meaning that there is no proof for intrauterine transmission of COVID-19 [19]. It is not yet known if there is a vertical transmission of SARS-CoV-2 through mother's milk during breastfeeding [20].

In addition, scientific studies have shown that SARSCoV-2 maintains its infectivity in suspensions for up to nine days and in the dry state it survives between 24 hours and nine days [21]. For all these reasons, all fresh biological samples must be considered as potentially infectious.

\section{ㅁ Which are the SARS-CoV-2 contaminated surfaces in a hospital?}

Surfaces in isolation rooms and even clinician shoe covers have tested positive as well. Moreover, the virus can spread via aerosols and fomites, and survive as aerosol for at least three hours and on surfaces for days [22, 23].

The virus can persist on surfaces like metal, glass, or plastic for up to a couple of days. Therefore, contaminated surfaces are frequently contacted in healthcare settings are a potential source of this new coronavirus transmission $[24,25]$. It is therefore necessary to implement protective measures also for the operating room, the most important being the medical staff protection, who should wear personal protective equipment (PPE) (Figure 4, a-d).

\section{a What is known so far about how to inactivate the virus?}

Although few things are known about this new coronavirus, it is considered that SARS-CoV-2 may be susceptible to disinfectants with proven activity against encapsulated viruses, such as sodium hypochlorite $(0.1 \%)$, for disinfecting surfaces in general, and (1\%), for disinfecting surfaces with blood); ethanol in a 
concentration ranging from $62 \%$ to $71 \% ; 0.5 \%$ hydrogen peroxide; quaternary ammonium compounds; and phenolic

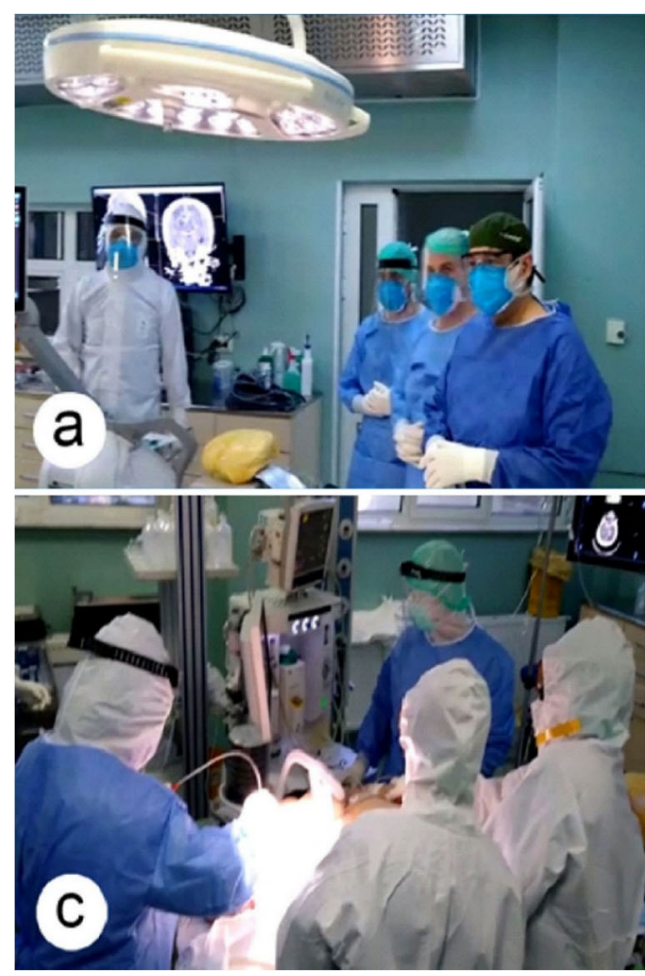

compounds, in accordance with the manufacturer's recommendations $[26,27]$.

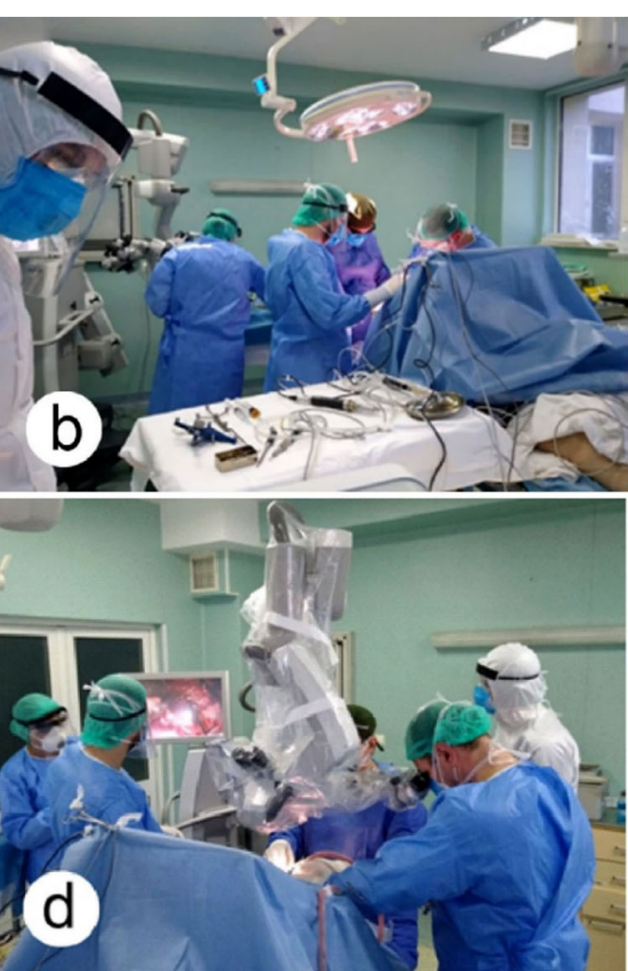

Figure 4 - (a-d) Specimen with SARS-CoV-2 infection are sampling in the operating room by neurosurgeon using protective measure (authors' collection).

There are scientific studies that have shown that formalin inactivates the coronaviruses in a time and temperature dependent manner, as follows: at $4^{\circ} \mathrm{C}$, the effect of formalin is inhibited; at $37^{\circ} \mathrm{C}$ or room temperature, formalin decreases the infectivity of the coronavirus after 24 hours; and at $56^{\circ} \mathrm{C}$ the coronavirus is thermally inactivated after 90 minutes $[28,29]$. At the same time, there are other published articles that have shown the thermal inactivation of coronavirus at $56^{\circ} \mathrm{C}$ in the absence of proteins; however, in the presence of a concentration of at least $20 \%$ protein in the medium, as is the case with liquids and surgical samples, thermal inactivation is less effective, so that a residual infectivity is maintained. For inactivation of the coronavirus, either a heat treatment at $67^{\circ} \mathrm{C}$ for 60 minutes can be used, as well as chemical substances, such as formalin, ethanol (with a concentration of at least $75 \%$ ) and acetone, which are all used as fixatives in the HP technique $[1,30]$.

Ultraviolet (UV) irradiation for 15 minutes has also been shown to reduce the infectivity of the new coronavirus, but in a way depending on the type of wave. UVC effectively inactivates SARS-CoV-2 in 40 minutes, while UVA requires the addition of psoralen to enhance virus inactivation. The presence of bovine serum albumin limits the ability of UVC and UVA to inactivate SARS-CoV-2 [28].

\section{ㅁ Preparing the laboratory of pathology for the activity carried out during the pandemic}

Fresh specimens from suspected or confirmed SARSCoV-2 patients are handled in the laboratory as smears or frozen section examinations may be requested. Chen \& Chi [30], two authors from Taiwan, propose a stepwise biosafety strategy for pathology laboratory, based on WHO and Centers for Disease Control and Prevention (CDC) Guidelines that include a combination of risk assessment, staff education, appropriate supply, sample transportation, handling and processing, and environmental protection [31].

According to $C D C$ General Guidance, all laboratories of pathology should perform a site-specific and activityspecific risk assessment to identify and mitigate risks (Table 1).

Table 1 - Site-specific and activity-specific risk assessment to prevent SARS-CoV-2 infection for

Laboratory of Pathology (according to [34, 36, 37])

Identification of laboratory activities that may be most exposed to risks:

- Extemporaneous procedure by smear preparation;

- Extemporaneous procedure by frozen section: laying the

slice on the freezing device; sample slicing in a cryostat; spreading of frozen slice on a slide;

- Centrifuged liquid-based cytology with smear preparation. Identification of working equipment that can ensure safety in the handling of infected samples.

Identification of staff potential exposure by recognition of:

- Type of specimen that could transmit the disease: liquids and fresh samples;

- Mode of transmission: aerosols and direct contact.

Establishing a working protocol for each laboratory procedure in order to reduce or eliminate steps that could lead to aerosol or droplets formation.

Performing the risk assessments of samples received in the laboratory by reviewing the clinical data on the request form, information obtained from the patient's physician, laboratory records and epidemiological data.

SARS-CoV-2: Severe acute respiratory syndrome coronavirus 2 . 
The laboratory director should analyze laboratory activities that may be most exposed to risks, working equipment that can ensure laboratory biosafety, the type of specimen that could transmit the disease, the mode of SARS-CoV-2 transmission. Also, a proper working protocol for each procedure involving laboratory staff should be realized (Tables 2 and 3 ).

Table 2 - Evaluation of the equipment and facilities required for the laboratory activity during the SARSCoV-2 pandemic (according to [34, 36, 37])

Centrifuge with sealed rotor or safety cups.

Cryostat where aerosols can be contained.

Certified BSC

Germicidal UVC lamp.

Adequate supply of specimen collection devices.

Appropriate disinfection materials: sodium hypochlorite $(0.1 \%)$, ethanol $(62 \%$ to $71 \%), 0.5 \%$ hydrogen peroxide, quaternary ammonium compounds and phenolic compounds).

Adequate supply of appropriate PPE: googles or disposable full-face shield, medical mask or approved N95 filtering face piece, two pairs of clean, nonsterile gloves, gown with long sleeves, apron.

Adequate supply for waste collection.

BSC: Biosafety cabinet; PPE: Personal protective equipment; SARSCoV-2: Severe acute respiratory syndrome coronavirus 2; UVC: Ultraviolet C.

Table 3 - Education of the laboratory personnel regarding the infection with SARS-CoV-2 (according to $[34,36,37])$

The competence and experience of laboratory staff must be established.

Permanent appropriate training program on infection prevention and control.

Permanent appropriate training program on basic GMPP

Permanent discussion with the laboratory work team in order to coordinate the activity in the sector.

Staff working in each sector of the laboratory must be scheduled.

Reorganization of staff working time to avoid congestion at work.

Permanently, all staff must be informed about the evolution of the pandemic in the country and abroad.

Permanently, all staff must be informed about the latest scientific acquisitions in the field of COVID-19 management in the laboratory of pathology.

Permanently, all staff must be informed about the policy of the hospital in which they work regarding the management of COVID-19 and to inform the superiors of the laboratory of any change.

Permanently, all staff must maintain ongoing virtual communication with colleagues in the lab, in the hospital and in other hospitals, including those working from home. All the staff should report any symptoms related with a possible viral infection: fever, tiredness, dry cough, chest pains, nasal congestion, runny nose, sore throat, and diarrhea.

All staff should perform hand hygiene frequently with soap and water.

The laboratory personnel should not create panic among colleagues but must be aware of the risks.

Laboratory staff must not spread rumors or false information inside the working team but should take all the needed information only from reliable sources.

COVID-19: Coronavirus disease 2019; GMPP: Good microbiological practices and procedures; SARS-CoV-2: Severe acute respiratory syndrome coronavirus 2 .

During the SARS-CoV-2 pandemic, the laboratory director should realize an evaluation of the equipment and facilities which exist already in the laboratory or which should be purchased in the future for the laboratory activity, i.e., centrifuge with sealed rotor or safety cups, cryostat, certified biosafety cabinet (BSC), germicidal UVC lamp, adequate specimen collection devices, disinfection materials (hands disinfectants and cleaning supplies), PPE (masks, gloves, goggles and face shields, long sleeves disposable gowns, waterproof aprons, waterproof gowns, disposable cap, shoe covers), and devices for waste collection (Table 2).

The $W H O$ also recommends that the laboratory director should ensure that staff are educated on infection prevention and control, and on updates about COVID-19 infection, but at the same time he/she must control if all laboratory professionals follow basic good microbiological practices and procedures (GMPP) for laboratory biosafety (Table 3).

Meantime, with the help of the hospital manager, the laboratory director should provide to the laboratory personnel counseling resources, encouraging them to report any symptom that could represent the infection with SARS-CoV-2: high fever, chills, cough, and shortness of breath or difficulty in breathing, but also some other symptoms, such as diarrhea, myalgia, fatigue, expectoration, and hemoptysis [27].

In the laboratory of pathology should be implemented a good communication within own staff and with the healthcare team (Table 4). Regarding the environmental protection, cleaning, and disinfection of surfaces to ensure effective infection prevention and control is required.

Table 4 - Communication within laboratory and with healthcare team (according to [34, 36, 37])

Virtual lines of communication must be opened between laboratory staff and all the medical teams from the hospital. For each request for an extemporaneous or cytopathological examination, a discussion should be held with the operating team to assess the case and determine the need to request and perform the examination, in order to avoid unnecessary exposure of laboratory staff.

Laboratory staff should collaborate with the hospital administration and with local Public Health Department.

\section{ㅁ Recommendations and guidance on safe handling and processing of surgical specimens with suspected or confirmed SARS-CoV-2 infection in the operating room and laboratory of pathology}

The handling and processing of biological samples from suspected or confirmed patients with SARS-CoV-2 infection must follow the Guidelines of $W H O$, United States $C D C, E C D C$ [26, 28, 31]. These regulations have been implemented in our hospital and have been customized because samples and liquids other than respiratory ones are handled. In what will follow, we present our experience.

\section{Safety procedures for transportation of a suspected or confirmed COVID-19 specimen}

Based on $W H O$ recommendations, body fluids or fresh tissue samples from suspected or confirmed COVID-19 positive patients should be transported as "UN3373 Biological Substance Category B" because this virus has high infectivity $[31,32]$. The packaging for "UN3373" consists of three containers: $(i)$ a leak-proof primary 
receptacle, (ii) a leak-proof secondary packaging, and (iii) an outer packaging of adequate strength. In our hospital, we consider that due to the properly disinfection of the first container inside the operating room by nebulization, and then the proper disinfection of the inside and outside of the second container with proper disinfectants, only two containers can be enough for the safety of the laboratory personnel.

But liquids and surgical samples taken for extemporaneous examination can be infected even though the patient is asymptomatic. As a preventive measure, in our hospital surgical specimens, even though the patient has one negative PCR test for COVID-19, are transported in two containers (Figure 5). The first is a plastic container, which should be well sealed and correctly labeled (we mark it as R1). Then, R1 is placed inside the second plastic container (we mark it as R2), which will be disinfected by spraying an adequate disinfectant both inside and outside the plastic box. The operating room staff must complete the sample accompanying form, noting whether or not the patient is suspected or confirmed with COVID-19. Before leaving the operating room, the person who transports the specimen should notify the laboratory by direct communication that the specimen will be transported (Table 5).

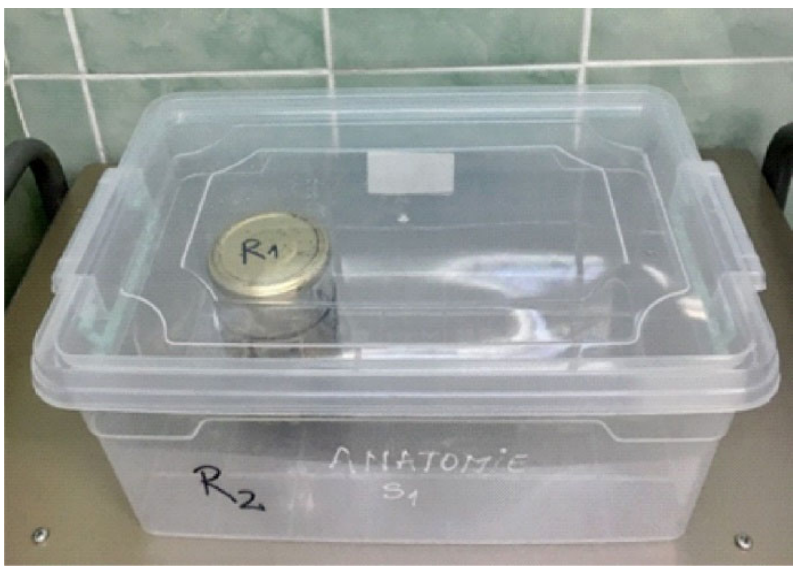

Figure 5 - Adequate specimen transportation in two recipients (R1 and R2) (authors' collection).

Table 5 - Transportation of SARS-CoV-2 infected surgical specimens

Surgical specimens from suspected or confirmed cases should be transported as "Biological Substance Category B", i.e., in two containers for the safety of all medical staff.

All specimens should be delivered by hand and correctly labeled.

Operating room personnel who transport surgical specimens should be well trained in safe handling practices and spill decontamination procedures.

The laboratory personnel should be notifying in a timely manner that the specimen is being transported.

SARS-CoV-2: Severe acute respiratory syndrome coronavirus 2 .

If the patient is COVID-19 positive and the extemporaneous (smear or frozen section) examination is not indicated because there is no BSC, the sample should be placed inside the R1 container, which contains $10 \%$ formalin, in a volume 20 times greater than the volume of the specimen. After nebulization of the operating room, personnel must properly seal and label the R1 primary container, mentioning the suspicion/confirmation of
SARS-CoV-2 infection, decontaminate it by spraying with a proper disinfectant and place it in the R2 transport plastic box. Before closing R2, it will be sprayed with disinfectant both inside and outside and then will be stored in a dedicated space within the operating room. All the above-mentioned procedures must be performed in a manner that minimizes the generation of aerosols and droplets.

Operating room staff should complete the sample accompanying form, noting whether the patient is suspected or confirmed with COVID-19.

After 24 hours from the surgery, the operating room staff will notify the laboratory about sending specimens from a patient with suspected or confirmed COVID-19, by direct communication. All biological samples must be transport to the laboratory by a person who is well trained in safe handling practices and spill decontamination procedures.

\section{Safety procedures for handling and processing a suspected or confirmed COVID-19 specimen in the laboratory}

In SARS-CoV-2 pandemic, cytology and other laboratory activities, such as pathological examination and processing of formalin-fixed or otherwise inactivated tissues (inclusion in paraffin block), routine staining and microscopic analysis of fixed smears are assigned to biosafety level 2 (BSL2) (Figure 6, a and b). Body fluids and tissue samples collected in the operating room and brought to the laboratory for extemporaneous diagnosis and cytopathological diagnosis, respectively, are very infectious, because these samples come fresh, without any prior fixation $[9,31]$.

In the laboratories of cytology and histopathology, special precautions must be taken when handling and preparing samples, as the steps of the techniques may lead to the formation of aerosols or droplets [9, 32-35]. Handling of biological samples from patients admitted during the SARS-CoV-2 pandemic should be performed properly in the BSC by personnel with demonstrated ability, as aerosols, droplets or splashes may occur during working with these samples.

\section{Specific procedures for cytopathology activity}

The $W H O$ has recommended an advanced biosafety policy in the cytopathology sector to prevent infections because until now it is unknown whether fixatives used in cytopathology as they are alcohol solutions with lower concentrations, could adequately inactivate for SARSCoV-2 [36].

However, the entire scientific community believes that processing of body fluids and fresh samples from inpatients during the SARS-CoV-2 pandemic is potentially infectious because these specimens contain or may contain HG3 biological agent. As such, because the cytopathology steps consist in manual decapping, splitting or diluting samples, vortexing, centrifuging, pipetting, mixing, and preparation for staining on, smears, initial processing (before inactivation) of all specimens should take place in a validated BSC or in a negative pressure room with at least 12 air changes per hour $[32,35]$. 




Figure 6 - (a and b) Certified biosafety cabinet (BSC) during working and during its disinfection with ultraviolet $C$ (UVC) light (authors' collection).

Centrifugation of suspected or confirmed COVID-19 patient's liquids must be performed using sealed centrifuge rotors (Figure 7) or sample cups which are loaded and unloaded in a BSC. However, all the steps of cytopathological procedure should be done by technicians with proven ability, as splashes, droplets or aerosols from these samples may occur during working with (when expulsing the aspirates from the needle or syringe, when the aspiration material is smeared and then slides are shaken through the air for drying, when loading and unloading the centrifuge tubes, when vigorously stirring or mixing liquids, when opening the liquid containers by removing the tube caps as these recipients can have a pressure different from the ambient pressure, when removing the supernatant from centrifuge tubes) (Table 6) $[9,31]$.

Because viral nucleic acids have been detected in different types of clinical samples, when handling and processing COVID-19 suspected or confirmed cytopathological specimens in a BSC, laboratory technicians should wear appropriate PPE (Table 2) (face mask, goggles, two pairs of gloves for a higher level of protection, longsleeved, water-resistant gowns or waterproof aprons, disposable cap) (Figure 8). The PPE must be used and handled in a correct manner, both when dressing and when undressing [31, 32]. All technical procedures must be performed in a manner that minimizes the generation of aerosols and droplets [9]. However, the number of individuals working in the procedure room should be kept to a minimum.

Before being brought to the pathologist for cytological examination, the slides must be disinfected with chloramine or another disinfectant and then placed on the slide tray.

The pathologists should use gloves when manipulating the cytological slides, since glass slides are touched by multiple hands until they reach the pathologist's desk and cannot be easily decontaminated. It can be used for disinfection the method of dipping the slides in $95 \%$ alcohol (or similar) for a couple of minutes or other surface disinfectants, especially ones with short contact times [36].



Figure 7 - Centrifuge with sealed rotor or safety cups (authors' collection).

Table 6 - Handling and processing the suspected and confirmed SARS-CoV-2 surgical samples and liquids (according to [31])

The laboratory staff should wear the PPE (medical masks, eye protection, long-sleeved, water-resistant gowns or waterproof aprons, and gloves).

Initial processing of all specimens should take place in a validated BSC.

In case of cytopathology procedure, implying manual decapping, splitting or diluting samples, vortexing, centrifuging, pipetting, mixing, and staining on smears should be conducted in a BSC

All technical procedures should be performed in a way that minimizes the generation of aerosols and droplets.

Processing specimens by methods that may lead to aerosols or droplets (including manual decapping, splitting or diluting samples, vortexing, centrifuging, pipetting, mixing, and staining on smears and air-drying or heat fixing smears) should be conducted in a class II BSC, according to $\mathrm{CDC} / \mathrm{WHO}$

Guidelines.

Appropriate disinfectants with proven activity against enveloped viruses should be used before and after samples processing.

The laboratory staff should perform hand hygiene with soap and water after samples processing.

The laboratory staff should clean and disinfect contaminated surfaces with effective disinfectants.

Contaminated equipment must be disposed as biohazardous waste.

BSC: Biosafety cabinet; CDC: Centers for Disease Control and Prevention and Control; PPE: Personal protective equipment; SARSCoV-2: Severe acute respiratory syndrome coronavirus 2; WHO: World Health Organization. 


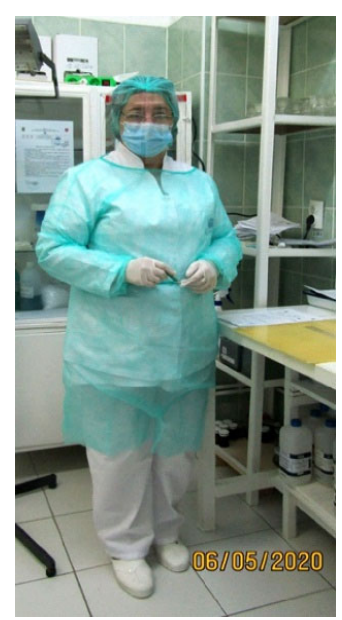

Figure 8-Personal protective equipment (PPE) (masks, gloves, goggles and face shields, long sleeves, disposable gowns, waterproof aprons, waterproof gowns, disposable cap) (authors' collection).

When a certified BSC is not available or the centrifuge with safety cups or sealed rotors cannot be used inside a BSC, laboratory technicians should use additional protection, such as a filtering facepiece 2 (FFP2) mask or N95 respirator, waterproof suit, a face shield, and other suitable protective equipment [37].

But some other authors consider that in a pandemic with a respiratory transmission virus, as SARS-CoV-2, if the microbiological BSC is not available in the laboratory, it is safer to suspend cytopathology activity, providing protection for laboratory staff.

\section{Specific procedures for frozen section examination}

In a similar manner with the handling of biological fluids, it seems more prudent not to perform frozen sections for suspected and confirmed COVID-19 cases [9] if the laboratory of pathology does not have appropriate conditions (BSC and proper PPE). However, in well-equipped laboratories, the processing of frozen sections from a confirmed COVID-19 patient should be performed by well-trained technicians, who wear appropriate PPE and use a cryostat where aerosols can be contained [37, 38]
(Figure 9, a and b). However, the same protective steps should be followed as in the case of cytopathology procedure.

\section{Specific procedures for HP technique}

Due to high infectivity of SARS-CoV-2, it appears prudent to refrain from performing sampling on fresh surgical samples or partially fixed specimens [9]. Because the HP procedures consist in fixation of tissue sample with formalin and then its infiltration with paraffin at high temperatures $\left(56-60^{\circ} \mathrm{C}\right)$, both procedures being considered to inactivate the coronavirus, some authors affirm that paraffin blocks should be categorized into the low-risk group of SARS-CoV-2 infectivity [31], but we appreciate that it is necessary to disinfect the samples before they are processed by the HP technique because they are manipulated by the laboratory staff before being introduced to the thermostat.

We propose the following disinfection scheme that corresponds to the inactivation times of the virus under the action of UV light, formalin, and temperature. Before sample arriving, laboratory personnel must be prepared. The staff should wear appropriate PPE that includes: surgical mask, goggles, gloves, lab coat, disposable gown, and disposable cap. Upon arrival in the laboratory of a fixed surgical specimen from the operating room, the laboratory technician opens the R2 transport box, disinfects its interior using a disinfectant with proven activity against enveloped viruses, then takes the R1 container and decontaminates it with UV lamp for 30 minutes (Figure 10). Then, he/she introduces R1 container into the thermostat of $37^{\circ} \mathrm{C}$, letting it there for 24 hours. The next day, the R1 container will be passed into the $56^{\circ} \mathrm{C}$ thermostat (Figure 10), letting it there for another 24 hours, after which the sample will be processed according to the classic HP technique, because it can be considered as having an absent or a very low risk of infectivity.

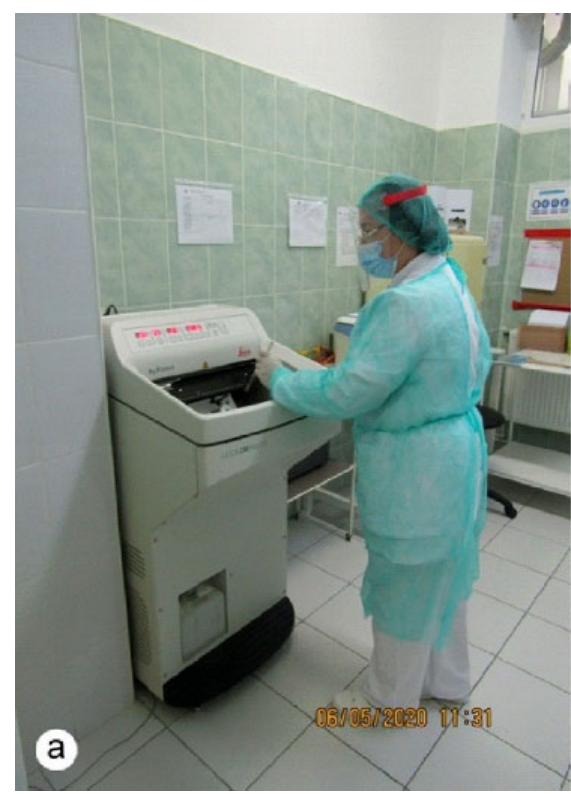

Figure 9 - (a and b) Frozen section can be done using a cryostat with UVC disinfection (authors' collection).



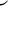

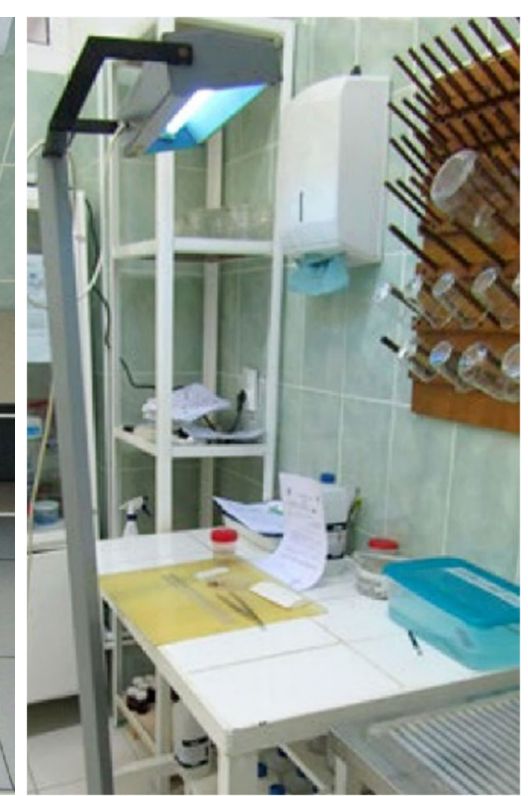

Figure 10 - Surgical sample is decontaminated with $U$ V lamp for 30 minutes (authors' collection). 


\section{$\neg$ Disinfection measures in the laboratory of pathology}

Because the virus can remain viable on surfaces (especially plastic and stainless-steel surfaces) for up to 72 hours, all laboratory staff will perform frequent decontamination of all working surfaces several times a day, using disinfectants with proven activity against enveloped viruses and detergents provided by the hospital. Also, it is required a daily cleaning of floors, chairs, handles, switches, technical equipment of the laboratory, and toilets, using appropriate disinfectants. It is essential to realize, daily or whenever it is necessary, disinfection of computer keyboards and phones, as well as frequently all touched areas of the microscopes.

Disinfectants and detergents should be used according to the manufacturer's recommendations. Particular attention should be paid not only to the selection of the proper disinfectant, but also to the contact time with the contaminated surface, which can range from 30 seconds to 10 minutes, to the dilution and the validity of the working solution.

After finishing all the steps of cytological and/or HP procedures and PPE removal, hand hygiene (with soap and water) should be performed carefully.

For the final disinfection of the sampling room space, 30 minutes before the end of the program, the technician should open the ultraviolet lamp and take care to close it at departure.

\section{ㅁ Conclusions}

In this pandemic period, laboratory personnel should be aware that any specimen received in the laboratory, might it be a body fluid or a surgical sample, may contain SARS-CoV-2 and should act after new working procedures. Also, laboratory personnel should pay attention to instruments, technical equipment, or environmental surfaces as these also can be contaminated with the new coronavirus. As such, the SARS-CoV-2 pandemic has brought new challenges and concerns about the activity of the laboratory of cytology and/or pathology. Because SARS-CoV2 is a HG3 pathogen, GMPP must be followed both in the processing of biological samples and in the protection of laboratory staff and of the environment. In order to ensure the safety of laboratory personnel during COVID-19 pandemic, as well as SARS-CoV-2 future outbreaks, we support protective measures regarding personnel equipment and special measures for disinfection of laboratory surfaces, technical equipment and instruments. In addition, we recommend restraint from performing extemporaneous examination (smear and frozen section) and cytopathological examination in laboratories that do not have adequate conditions for handling and processing HG3 pathogens.

\section{Conflict of interests}

The authors declare that they have no conflict of interests.

\section{References}

[1] Rabenau HF, Cinatl J, Morgenstern B, Bauer G, Preiser W, Doerr HW. Stability and inactivation of SARS coronavirus.
Med Microbiol Immunol, 2005, 194(1-2):1-6. https://doi.org/ 10.1007/s00430-004-0219-0 PMID: 15118911 PMCID: PMC 7086689

[2] World Health Organization (WHO). Middle East respiratory syndrome coronavirus (MERS-CoV). https://www.who.int/ emergencies/mers-cov/en/

[3] Chang L, Yan Y, Wang L. Coronavirus disease 2019: coronaviruses and blood safety. Transfus Med Rev, 2020, 34(2): 75-80. https://doi.org/10.1016/j.tmrv.2020.02.003 PMID: 32107119 PMCID: PMC7135848

[4] Guarner J. Three emerging coronaviruses in two decades: the story of SARS, MERS, and now COVID-19. Am J Clin Pathol, 2020, 153(4):420-421. https://doi.org/10.1093/ajcp/ aqaa029 PMID: 32053148 PMCID: PMC7109697

[5] Yi Y, Lagniton PNP, Ye S, Li E, Xu RH. COVID-19: what has been learned and to be learned about the novel coronavirus disease. Int J Biol Sci, 2020, 16(10):1753-1766. https://doi. org/10.7150/ijbs.45134 PMID: 32226295 PMCID: PMC7098028

[6] Peeri NC, Shrestha N, Rahman MS, Zaki R, Tan Z, Bibi S, Baghbanzadeh M, Aghamohammadi N, Zhang W, Haque U. The SARS, MERS and novel coronavirus (COVID-19) epidemics, the newest and biggest global health threats: what lessons have we learned? Int J Epidemiol, 2020, 49(3):717-726. https://doi.org/10.1093/ije/dyaa033 PMID: 32086938 PMCID: PMC7197734

[7] Public Health England. COVID-19: safe handling and processing for samples in laboratories. COVID-19: Guidance for Sampling and for Diagnostic Laboratories, updated: 16 July 2020. https:// www.gov.uk/government/publications/wuhan-novel-corona virus-guidance-for-clinical-diagnostic-laboratories/wuhannovel-coronavirus-handling-and-processing-of-laboratoryspecimens

[8] Arbeitsschutzausschüsse. Begründung zur vorläufigen Einstufung des Virus SARS-CoV-2 in Risikogruppe 3 und Empfehlungen zu nicht gezielten Tätigkeiten (Labordiagnostik) und gezielten Tätigkeiten mit SARS-CoV-2. Beschluss 1/2020 des Ausschuss für Biologische Arbeitsstoffe (ABAS) vom 19.2.2020, 1-3. https://www.arbeitsschutz.sachsen.de/download/Beschluss des_ABAS_zur_Einstufung_SARS_CoV_2.pdf

[9] Ödega KI, Iyamah E, Ibadin EE, Idomeh FĀ, Odega DE. Safe laboratory practices in the light of COVID-19 pandemic: way forward in a resource limited setting. Preprints, 2020, 2020040103. https://doi.org/10.20944/preprints202004.0103.v1

[10] European Centre for Disease Prevention and Control (ECDC). Novel coronavirus disease 2019 (COVID-19) pandemic: increased transmission in the EU/EEA and the UK - sixth update. Rapid Risk Assessment, ECDC, Stockholm, 12 March 2020, 1-28. https://www.ecdc.europa.eu/sites/default/files/ documents/RRA-sixth-update-Outbreak-of-novel-coronavirusdisease-2019-COVID-19.pdf

[11] Jiehao C, Jin X, Daojiong L, Zhi Y, Lei X, Zhenghai Q, Yuehua $Z$, Hua Z, Ran J, Pengcheng L, Xiangshi W, Yanling G, Aimei X, He T, Hailing C, Chuning W, Jingjing L, Jianshe W, Mei Z. A case series of children with 2019 novel coronavirus infection: clinical and epidemiological features. Clin Infect Dis, 2020, 71(6):1547-1551. https://doi.org/10. 1093/cid/ciaa198 PMID: 32112072 PMCID: PMC7108143

[12] ECDC. Novel coronavirus (SARS-CoV-2). Discharge criteria for confirmed COVID-19 cases - when is it safe to discharge COVID-19 cases from the hospital or end home isolation? ECDC Technical Report, 2020, 1-5. https://www.ecdc.europa. eu/sites/defaultffiles/documents/COVID-19-Discharge-criteria.pdf

[13] Yang P, Wang X. COVID-19: a new challenge for human beings. Cell Mol Immunol, 2020, 17(5):555-557. https://doi. org/10.1038/s41423-020-0407-x PMID: 32235915 PMCID: PMC7110263

[14] Wang W, Xu Y, Gao R, Lu R, Han K, Wu G, Tan W. Detection of SARS-CoV-2 in different types of clinical specimens. JAMA, 2020, 323(18):1843-1844. https://doi.org/10.1001/jama.2020. 3786 PMID: 32159775 PMCID: PMC7066521

[15] Peng L, Liu J, Xu W, Luo Q, Chen D, Lei Z, Huang Z, Li X, Deng K, Lin B, Gao Z. SARS-CoV-2 can be detected in urine, blood, anal swabs and oropharyngeal swabs specimens. J Med Virol, 2020, 92(9):1676-1680. https://doi.org/10.1002/ jmv.25936 PMID: 32330305 PMCID: PMC7264521

[16] To KKW, Tsang OTY, Yip CCY, Chan KH, Wu TC, Chan JMC, Leung WS, Chik TSH, Choi CYC, Kandamby DH, Lung DC, 
Tam AR, Poon RWS, Fung AYF, Hung IFN, Cheng VCC, Chan JFW, Yuen KY. Consistent detection of 2019 novel coronavirus in saliva. Clin Infect Dis, 2020, 71(15):841-843. https://doi.org/10.1093/cid/ciaa149 PMID: 32047895 PMCID: PMC7108139

[17] Salehi S, Abedi A, Balakrishnan S, Gholamrezanezhad A. Coronavirus disease 2019 (COVID-19): a systematic review of imaging findings in 919 patients. AJR Am J Roentgenol, 2020, 215(1):87-93. https://doi.org/10.2214/AJR.20.23034 PMID: 32174129

[18] Guan WJ, Ni ZY, Hu Y, Liang WH, Ou CQ, He JX, Liu L, Shan H, Lei CL, Hui DSC, Du B, Li LJ, Zeng G, Yuen KY, Chen RC, Tang CL, Wang T, Chen PY, Xiang J, Li SY, Wang JL, Liang ZJ, Peng YX, Wei L, Liu Y, Hu YH, Peng P, Wang JM, Liu JY, Chen Z, Li G, Zheng ZJ, Qiu SQ, Luo J, Ye CJ, Zhu SY, Zhong NS; China Medical Treatment Expert Group for Covid-19. Clinical characteristics of coronavirus disease 2019 in China. N Engl J Med, 2020, 382(18):17081720. https://doi.org/10.1056/NEJMoa2002032 PMID: 32109013 PMCID: PMC7092819

[19] Karimi-Zarchi M, Neamatzadeh H, Dastgheib SA, Abbasi $\mathrm{H}$, Mirjalili SR, Behforouz A, Ferdosian F, Bahrami R. Vertical transmission of coronavirus disease 19 (COVID-19) from infected pregnant mothers to neonates: a review. Fetal Pediatr Pathol, 2020, 39(3):246-250. https://doi.org/10.1080/15513 815.2020.1747120 PMID: 32238084 PMCID: PMC7157948

[20] Marinelli KA. International perspectives concerning donor milk banking during the SARS-CoV-2 (COVID-19) pandemic. J Hum Lact, 2020, 36(3):492-497. https://doi.org/10.1177/ 0890334420917661 PMID: 32223581 PMCID: PMC7201197

[21] Pal M, Berhanu G, Desalegn C, Kandi V. Severe acute respiratory syndrome coronavirus-2 (SARS-CoV-2): an update. Cureus, 2020, 12(3):e7423. https://doi.org/10.7759/cureus. 7423 PMID: 32337143 PMCID: PMC7182166

[22] Brat GA, Hersey SP, Chhabra K, Gupta A, Scott J. Protecting surgical teams during the COVID-19 outbreak: a narrative review and clinical considerations. Ann Surg, 2020 Apr 17, 10.1097/SLA.0000000000003926. https://doi.org/10.1097/SLA. 0000000000003926 PMID: 32379080 PMCID: PMC7224623

[23] Gu J, Han B, Wang J. COVID-19: gastrointestinal manifestations and potential fecal-oral transmission. Gastroenterology, 2020, 158(6):1518-1519. https://doi.org/10.1053/.gastro.2020. 02.054 PMID: 32142785 PMCID: PMC7130192

[24] Peng X, Xu X, Li Y, Cheng L, Zhou X, Ren B. Transmission routes of $2019-n C o V$ and controls in dental practice. Int J Oral Sci, 2020, 12(1):9. https://doi.org/10.1038/s41368-0200075-9 PMID: 32127517 PMCID: PMC7054527

[25] Kampf G, Todt D, Pfaender S, Steinmann E. Persistence of coronaviruses on inanimate surfaces and their inactivation with biocidal agents. J Hosp Infect, 2020, 104(3):246-251. https://doi.org/10.1016/j.jhin.2020.01.022 PMID: 32035997 PMCID: PMC7132493

[26] Darnell MER, Subbarao K, Feinstone SM, Taylor DR. Inactivation of the coronavirus that induces severe acute respiratory syndrome, SARS-CoV. J Virol Methods, 2004, 121(1):85-91. https://doi.org/10.1016/j.jviromet.2004.06.006 PMID: 15350737 PMCID: PMC7112912

[27] Darnell MER, Taylor DR. Evaluation of inactivation methods for severe acute respiratory syndrome coronavirus in noncellular blood products. Transfusion, 2006, 46(10):1770-1777. https:// doi.org/10.1111/j.1537-2995.2006.00976.x PMID: 17002634 PMCID: PMC7201869

[28] Duan SM, Zhao XS, Wen RF, Huang JJ, Pi GH, Zhang SX, Han J, Bi SL, Ruan L, Dong XP; SARS Research Team. Stability of SARS coronavirus in human specimens and environment and its sensitivity to heating and UV irradiation. Biomed Environ Sci, 2003, 16(3):246-255. PMID: 14631830

[29] Kariwa H, Fujii N, Takashima I. Inactivation of SARS coronavirus by means of povidone-iodine, physical conditions, and chemical reagents. Dermatology, 2006, 212(Suppl 1):119-123. https://doi.org/10.1159/000089211 PMID: 16490989 PMCID: PMC7179540

[30] Chen CC, Chi CY. Biosafety in the preparation and processing of cytology specimens with potential coronavirus (COVID-19) infection: perspectives from Taiwan. Cancer Cytopathol, 2020, 128(5):309-316. https://doi.org/10.1002/cncy.22280 PMID: 32259402 PMCID: PMC7262216

[31] Centers for Disease Control and Prevention (CDC). Coronavirus disease 2019. Lab Biosafety Guidelines, updated March 31, 2020. https://www.cdc.gov/coronavirus/2019-nCoV/lab/lab-bio safety-guidelines.html

[32] Public Health England. Guidance. COVID-19: safe handling and processing for samples in laboratories. Open Government License (OGL) ver. 3.0, updated 3 April 2020, 1-7. https:// allcatsrgrey.org.uk/wp/download/public_health/pathology/ COVID-19_-safe-handling-and-processing-for-samples-inlaboratories-GOV.UK_.pdf

[33] Pambuccian SE. The COVID-19 pandemic: implications for the cytology laboratory. J Am Soc Cytopathol, 2020, 9(3): 202-211. https://doi.org/10.1016/j.jasc.2020.03.001 PMID: 32284276 PMCID: PMC7104051

[34] Iwen PC, Stiles KL, Pentella MA. Safety considerations in the laboratory testing of specimens suspected or known to contain the severe acute respiratory syndrome coronavirus 2 (SARS-CoV-2). Am J Clin Pathol, 2020, 153(5):567-570. https://doi.org/10.1093/ajcp/aqaa047 PMID: 32190890 PMCID: PMC7184496

[35] Henwood AF. Coronavirus disinfection in histopathology. J Histotechnol, 2020, 43(2):102-104. https://doi.org/10.1080/ 01478885.2020.1734718 PMID: 32116147

[36] World Health Organization (WHO). Guidance on regulations for the transport of infectious substances 2019-2020. Cited January 28, 2020. https://www.who.int/ihr/publications/WHO -WHE-CPI-2019.20

[37] World Health Organization (WHO). Laboratory biosafety guidance related to the novel coronavirus (2019-nCoV). Interim Guidance, cited January 28, 2020, 1-12. https://www.who.int/docs/defaultsource/coronaviruse/laboratory-biosafety-novel-coronavirusversion-1-1.pdf

[38] Public Health England. COVID-19: guidance for sampling and for diagnostic laboratories. Information for clinical diagnostic laboratories regarding safety, sampling and packaging specimens associated with COVID-19. Coronavirus (COVID-19), Rules, Guidance and Support, published 15 January 2020. https:// www.gov.uk/government/publications/wuhan-novel-corona virus-guidance-for-clinical-diagnostic-laboratories

\section{Corresponding authors}

Claudia Florida Costea, Associate Professor, MD, PhD, Department of Surgery II, Faculty of Medicine, Grigore T. Popa University of Medicine and Pharmacy, 16 University Street, 700115 laşi, Romania; Phone +40744-972 648, e-mail: costea10@yahoo.com

Şerban Turliuc, Associate Professor, MD, PhD, Socola Institute of Psychiatry, 36 Bucium Highway, 700282 laşi, Romania; Phone +40745-203 002, e-mail: serban_turliuc@yahoo.com 